\title{
MicroRNA-154 functions as a tumor suppressor in osteosarcoma by targeting Wnt5a
}

\author{
HUI ZHOU ${ }^{1}$, MINGLEI ZHANG ${ }^{1}$, HONGPING YUAN ${ }^{2}$, WEI ZHENG ${ }^{2}$, \\ CHUNYAN MENG ${ }^{1}$ and DONGXU ZHAO ${ }^{1}$ \\ ${ }^{1}$ China-Japan Union Hospital of Jilin University, Nanguan, Changchun 13033; \\ ${ }^{2}$ The Fourth Hospital of Jilin University, Changchun, Jilin 130021, P.R. China
}

Received September 9, 2015; Accepted October 27, 2015

DOI: $10.3892 / o r .2015 .4495$

\begin{abstract}
MicroRNAs (miRNAs) are small non-coding RNAs that are involved in tumor initiation and development by suppressing target gene expression. miRNA-154 has been shown to be important in tumorigenesis in many types of cancers. However, its role in osteosarcoma (OS) remains unknown. In the present study, we focused on the roles and mechanisms of miR-154 in OS development. The results of quantitative RT-PCR showed that miR-154 expression was decreased in primary OS tumor samples and cell lines compared to levels in the matched adjacent normal tissues and human normal osteoblast cells (NHOst). Restoration of expression in U2OS cells inhibited cell proliferation, colony formation, migration and invasion, as well as induced cell cycle arrest at the G1 stage. Bioinformatic prediction suggested that Wnt5a is a target gene of miR-154. It was further verified that Wnt5a is a target gene of miR-150 in OS cells using luciferas assay, mRNA and protein expression analysis. Wnt5a was upregulated in OS cell lines and primary tumor samples, and its mRNA expression level was negatively correlated with the miR-154 level in the OS tissues. Restored expression of Wnt5a weakened miR-154-mediated suppression of tumor progression. Taken together, these findings suggest that miR-154 functions as a tumor suppressor in OS by partially suppressing Wnt5a expression.

Introduction
\end{abstract}

Osteosarcoma (OS) is a rare malignancy but the most common bone sarcoma affecting rapidly growing bones, particularly in children and adolescents (1). Along with the development of

Correspondence to: Dr Dongxu Zhao, China-Japan Union Hospital of Jilin University, 126 Xiantai Street, Economy Technical Developing Zone, Nanguan, Changchun 13033, P.R. China

E-mail: zhaodongxu909@sina.com

Key words: osteosarcoma, microRNAs, miR-154, Wnt5a therapeutic strategies combining chemotherapy, surgery and sometimes radiotherapy, the prognosis of OS has gradually improved over the past 30 years (2). However, for patients who present with metastatic disease or whose tumor recurs, the 5 -year survival is less than $30 \%$ (3). Although great efforts have been taken to explore the molecular mechanisms of the carcinogenesis of OS, the fundamental molecular mechanisms of OS underlying initiation and development have not been fully elucidated (4). This emphasizes the need for novel molecular targets and novel alternative therapeutic strategies to improve the clinical outcome of patients suffering OS.

$>$ MicroRNAs (miRNAs) are non-coding messenger RNA (mRNA) sequences containing approximately 22 nucleotides that act as important regulators of gene expression by specifically binding and cleaving mRNAs or inhibiting their translation $(3,5,6)$. Accumulating evidence has demonstrated that miRNAs are involved in various biological processes, such as cell proliferation, apoptosis, migration, invasion, differentiation, stress resistance, fat metabolism and development $(7,8)$. Approximately half of all human miRNAs are located in cancer-associated genomic regions; thus, they function as tumor-suppressor or oncogenic miRNAs by modification either of oncogenic or suppressor genes (9). In human OS, a number of miRNAs have been identified to be aberrantly overexpressed or downregulated during its progression, such as miR-34a, miR-125b, miR-143, miR-21, miR-503 and miR-217 (10-15). These miRNAs play oncogenic or tumor-suppressive roles in OS by suppressing their target genes.

miR-154 is located on human chromosome 14q32, which is a very conservative miRNA cluster in mammalians (16). Recently, several reports have demonstrated that miR-154 is a downregulated miRNA in prostate (17), breast (18), liver (17), non-small cell lung (19), colorectal (20) and thyroid cancer (21), suggesting that miR-154 plays a tumor-suppressor role. However, the functions and underlying molecular mechanisms of miR-154 in osteosarcoma are largely unknown. Therefore, the aims of this study were to investigate the expression of miR-150 in OS cell lines and primary tumor samples and assess its effects on cell proliferation, cell cycle distribution, apoptosis, migration and invasion, as well as to determine its target gene and molecular mechanism in OS cells. 


\section{Materials and methods}

Patients and tissue samples. Primary OS tissue samples and their corresponding adjacent normal bone tissues were obtained from 44 patients who underwent OS tissue resection at the China-Japan Union Hospital of Jilin University (Changchun, China) from September 2009 to March 2015, after receiving adequate patient informed consent. All human osteosarcoma biopsy specimens were obtained from primary lesions. The matched normal tissue samples adjacent to the tumor were obtained 5-cm distant from the peripheral tumor cells, which were further confirmed by pathologists. All patients did not undergo any therapy before recruitment to the present study. All tissues were immediately snap frozen in liquid nitrogen and stored at $-80^{\circ} \mathrm{C}$ until use. The study was approved by the Medical Ethics Committee of Jilin University (Changchun, China).

Cell lines and cell culture. Human osteosarcoma cell lines (HOS, Saos-2, U2OS and MG-63) and normal osteoblast cells (NHOst) were purchased from the Chinese Cell Bank of the Chinese Academy of Sciences (Shanghai, China), and were cultured in Dulbecco's modified Eagle's medium (DMEM; Invitrogen, Carlsbad, CA, USA) supplemented with $10 \%$ fetal bovine serum (Invitrogen) and streptomycin $(100 \mathrm{mg} / \mathrm{ml})$, and penicillin $(100 \mathrm{U} / \mathrm{ml})$ in a humidified incubator with $5 \% \mathrm{CO}_{2}$ at $37^{\circ} \mathrm{C}$.

$R N A$ extraction and quantitative reverse transcription-PCR Total RNA was extracted from cultured cells or tissues using TRIzol (Invitrogen) according to the manufacturer's protocol. For detection of the miR-154 level, cDNA was synthesized from $5 \mathrm{ng}$ of total RNA using the Taqman miRNA reverse transcription kit (Applied Biosystems, Foster City, CA, USA) The expression levels of miR-154 were quantified using the miRNA-specific TaqMan miRNA assay kit with miR-154-specific primers (both from Applied Biosystems) under the ABI 7500 sequence detection system (ABI-Prism; Applied Biosystems). For detection of the miR-154 level, cDNAs were synthesized from total RNA using the PrimeScript RT reagent kit (Takara, Dalian, China) according to the manufacturer's instructions. The following primers were used to amplify Wnt5a: sense primer, 5'-CTTCGCCCAGGTTGT AATTGAAGC-3 and antisense primer, 5'-CTGCCAAAAAC AGAGGTGTTATCC-3'. Glyceraldehyde-3-phosphate dehydrogenase (GAPDH) was amplified as an internal control using the sense primer, 5'-ACCACAGTCCATGCCATCAC-3' and the antisense primer, 5'-TCCACCACCCTGTTGCTG TA-3'. Quantitative reverse transcription-PCR (qRT-PCR) was performed using $\mathrm{SYBR}^{\circledR}$-Green PCR Master Mix on the ABI 7500 system. The relative level of Wnt5a was normalized with GAPDH, and miR-154 was normalized with U6 using the $2^{-\Delta \Delta \mathrm{Ct}}$ method.

Cell transfection. The miR-154 mimic or the corresponding negative control (miR-NC) were purchased from GenePharma (Shanghai, China) The Wnt5a overexpression plasmid (pCDNA3.1-Wnt5a) and blank vector pCDNA3.1 were a kind gift from Dr Yuyi Yao (Xuzhou Medical University). Transfection was performed in U2OS cells using
Lipofectamine 2000 (Invitrogen) according to the manufacturer's protocol. Transfection efficiencies were evaluated in every experiment $48 \mathrm{~h}$ post-transfection.

Cell proliferation and colony formation assay. The cell proliferation was determined by MTT assay. Briefly, U2OS cells $\left(5 \times 10^{3}\right.$ cells/well) were seeded into a 96 -well cell culture plate and underwent transfection. Four hours before the end of the experiment, $10 \mu 1$ of 3-(4,5-dimethylthiazol-2-yl)-2,5-diphenyl tetrazolium bromide (MTT) $(5 \mathrm{mg} / \mathrm{ml})$ was added, and the cells were incubated at $37^{\circ} \mathrm{C}$. Then, the medium was removed and the residue was dissolved in $150 \mu 1$ dimethyl sulfoxide (DMSO; Sigma-Aldrich). The absorbance of each well was read at $570 \mathrm{~nm}$ under a microplate reader (Molecular Devices, Menlo Park, CA, USA).

For the colony formation assay, transfected cells were digested and a single-cell suspension was prepared. Then, the cells were added to 6 -well plates $(1,000$ cells/well) followed by incubation under a normal condition for $24 \mathrm{~h}$. Non-adherent cells were removed. After culture for two weeks, the colonies were fixed with $4 \%$ paraformaldehyde for $20 \mathrm{~min}$ and counted after staining with $1 \%$ crystal violet. The percentage of colony formation was calculated by adjusting the control to $100 \%$.

Cell cycle analysis. Transfected cells were harvested using trypsinization, washed in ice-cold PBS, and fixed in ice-cold ethanol in PBS and incubated overnight at $-20^{\circ} \mathrm{C}$. The cells were pelleted and resuspended in $200 \mu \mathrm{l}$ of PBS with $50 \mu \mathrm{l}$ RNAase $\mathrm{A}$, and incubated at $37^{\circ} \mathrm{C}$ for $1 \mathrm{~h}$. Then, propidium iodide (PI, $750 \mu \mathrm{l}$ ) was added and incubated for $15 \mathrm{~min}$ at room temperature. The DNA contents of the samples were determined by FACSCalibur ${ }^{\mathrm{TM}}$ flow cytometer (BD Biosciences San Jose, CA, USA). The flow data were then analyzed by CellQuest software (BD Biosciences).

Cell migration and cell invasion. Cell migration and invasion were determined by a wound healing and invasion chamber assay, respectively. For the wound healing assay, transfected cells were seeded into 6-well tissue culture plates for $48 \mathrm{~h}$. Thereafter, an artificial homogenous wound was created in monolayer using a sterile plastic micropipette tip. After wounding, the debris was removed by washing the cells with serum-free medium. The cells were then cultured for another $24 \mathrm{~h}$ with serum-free medium. Images were captured at different time points $(0$ and $24 \mathrm{~h})$ under a light microscope (Leica DMR; Leica, Wetzlar, Germany). Individual cells were quantified as an average of at least five fields for each experiment.

For the invasion assays, transfected cells with $200 \mu$ of serum-free DMEM were placed into the upper chamber of an insert coated with Matrigel (BD Biosciences) following the manufacturer's protocol. DMEM containing 20\% FBS was added to the lower chamber as the chemoattractant. After $48 \mathrm{~h}$ of incubation, the cells remaining on the upper membrane were removed with cotton swabs, whereas those that had invaded through the membrane were fixed in $90 \%$ alcohol and stained with $0.1 \%$ crystal violet, and were photographed under an inverted microscope (magnification, x200; Olympus, Tokyo, Japan). The number of invaded cells was counted in five randomly selected fields. 

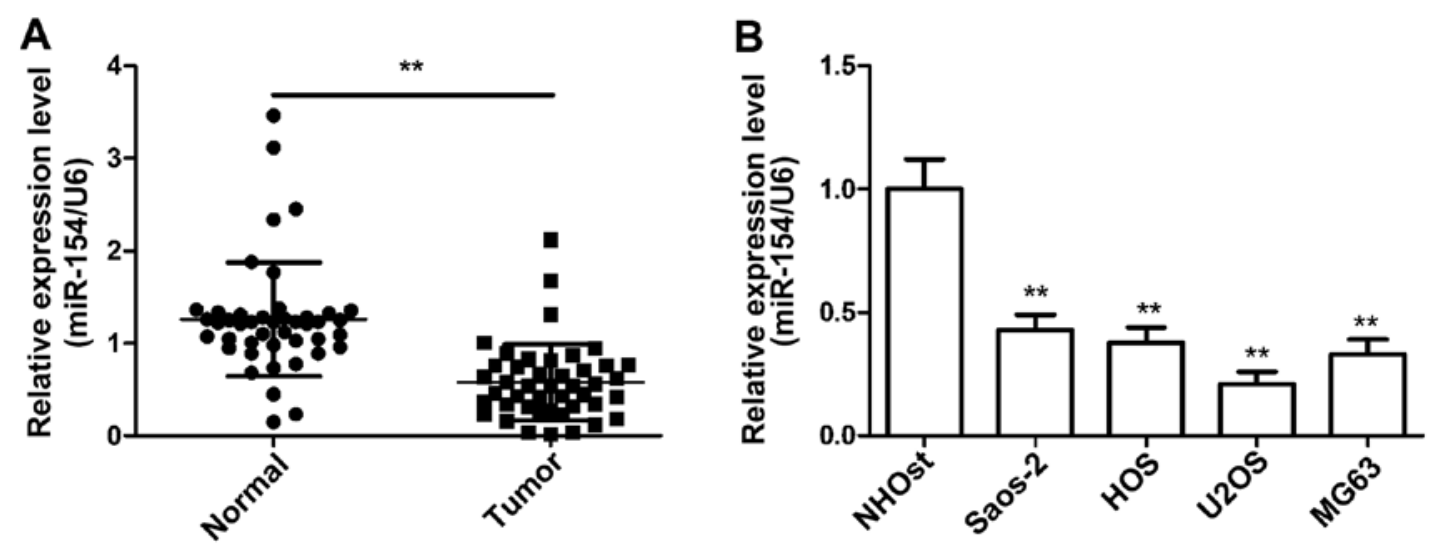

Figure 1. miR-154 expression is downregulated in osteosarcoma (OS) tissue samples and cell lines. (A) Quantitative RT-PCR (qRT-PCR) analysis of miR-154 expression in human OS tissue samples and their corresponding adjacent normal tissues from $44 \mathrm{OS}$ patients. ${ }^{* *} \mathrm{P}<0,01$ vs. nermal tissue. (B) qRT-PCR analysis of miR-154 expression in four OS cell lines (HOS, Saos-2, U2OS and MG-63) and normal osteoblast cells (NHOst).

Vector construction and luciferase assays. The wild-type 3'-UTR segment of Wnt5a containing the potential binding site of miR-154 was synthesized, annealed, and ligased into the pGL3-control vector (Ambion, Austin, TX, USA) at XhoI/NotI restriction sites (Promega, Madison, WI, USA). Mutations of Wnt5a 3'-UTR were introduced using the QuikChange site-directed mutagenesis kit (Stratagene, La Jolla, CA, USA), then ligased into the XhoI and NotI sites in the pGL3-control vector (Ambion).

For the luciferase assays, MG63 cells were co-transfected with wild-type (WT) or mutant (Mut) 3'-UTR of pGL3-Wnt5a and miR-154 or the miR-NC, and cultured for $48 \mathrm{~h}$. Then the dual luciferase activities were examined using the Dual-Luciferase Reporter Assay system(Promega).

Western blot analysis. Cells or tissues were harvested, and then the total protein was isolated with $\mathrm{M}-\mathrm{PER}^{\circledR}$ Mammahian protein extraction reagent (Plerce, Rockford, IL, USA) according to the manufacturer's protocol. Total protein concentrations were assessed using the BCA assay kit (Sigma). Samples with the same amount of total protein $(30 \mu \mathrm{g})$ were separated by $10 \%$ SDS-PAGE gel and transferred onto nitrocellulose membranes (Millipore, Boston, MA, USA). The membranes were blocked with TBST containing 5\% non-fat milk for $2 \mathrm{~h}$ and incubated with antibodies against human Wnt5a (1:1,500) and anti-GAPDH (1:3,000; both from Santa Cruz Biotechnology, Santa Cruz, CA, USA) at $4^{\circ} \mathrm{C}$ overnight. After washing with TBST twice, the membranes were then incubated with an HRP-conjugated goat anti-mouse IgG antibody (1:5,000, Santa Cruz Biotechnology) for $2 \mathrm{~h}$ at room temperature. Protein bands were visualized on X-ray film using an enhanced chemiluminescence detection system (ECL; Beyotime, Shanghai, China). GAPDH was used as an endogenous reference.

Statistical analysis. All data are presented as mean \pm standard deviation (SD) from at least three separate experiments. Data were analyzed using SPSS 16.0 (SPSS, Chicago, IL, USA). Statistical significance was evaluated using the Student's t-test or ANOVA. $\mathrm{P}<0.05$ was considered to indicate a statistically significant difference.

\begin{abstract}
Results
miR-154 is downregulated in osteosarcoma tissues and cell lines. To investigate the possible role of miR-154 in OS, we first examined the expression of miR-154 in 44 human OS specimens and corresponding adjacent normal tissues by qRT-PCR. As shown in Fig. 1A, the expression levels of miR-154 in the OS samples were lower than those in the normal tissue samples. Similarly, miR-154 expression was downregulated in the human OS cell lines (HOS, Saos-2, U2OS and MG-63) compared with the NHOst cells (Fig. 1B). U2OS cells exhibited the lowest expression of miR-154 among the four OS cell lines, and were selected for subsequent studies. These results provided us with initial evidence that miR-154 may be a tumor-suppressor miRNA in the development of human OS.
\end{abstract}

miR-154 inhibis the cell growth of osteosarcoma cells. To investigate the cellular function of miR-154 in OS, U2OS cells were transfected with the miR-154 mimic or miR-NC. qRT-PCR was used to verify the transfection effect. The miR-154 level was higher in the U2OS cells transfected with the miR-154 mimic than the expression level in the cells transfected with miR-NC (Fig. 2A). Cell proliferation and colony formation assay were then assessed in the U2OS cells transfected with miR-154 mimic or miR-NC. The data indicated that restoration of miR-154 expression in the U2OS cells significantly inhibited cell proliferation (Fig. 2B) and colony formation (Fig. 2C). As proliferation is directly linked to cell cycle distribution, the effect of miR-154 on cell cycle progression was analyzed. Compared with miR-NC, U2OS cells transfected with the miR-154 mimic displayed an increased percentage of cells in the G1 phase and fewer cells in the $\mathrm{S}$ phase (Fig. 2D). These results suggest that miR-154 inhibited OS cell growth partly due to G1-phase arrest.

miR-154 inhibits cell migration and invasion in osteosarcoma cells. To investigate the role of miR-154 in OS migration and invasion, U2OS cells were transfected with the miR-154 mimic, and then wound healing and invasion chamber assays were performed. It was found that upregulation of miR-154 signifi- 
A

C

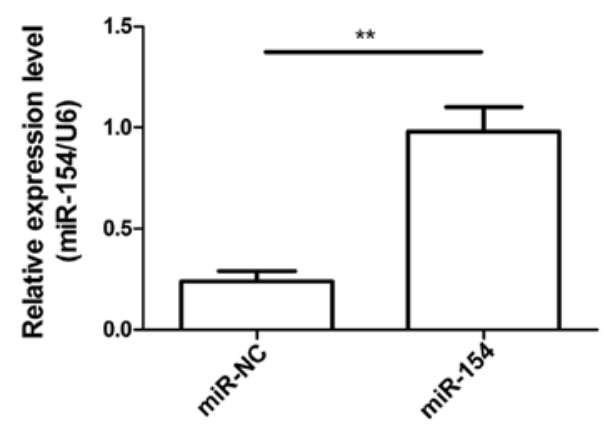

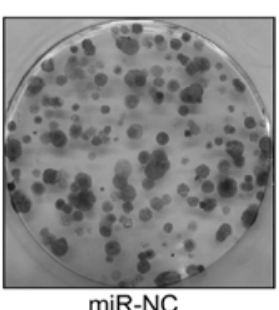

miR-NC

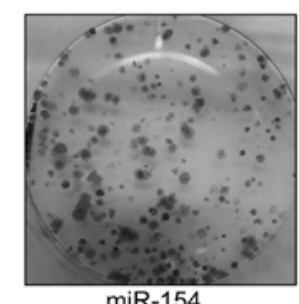

$\mathrm{D}$
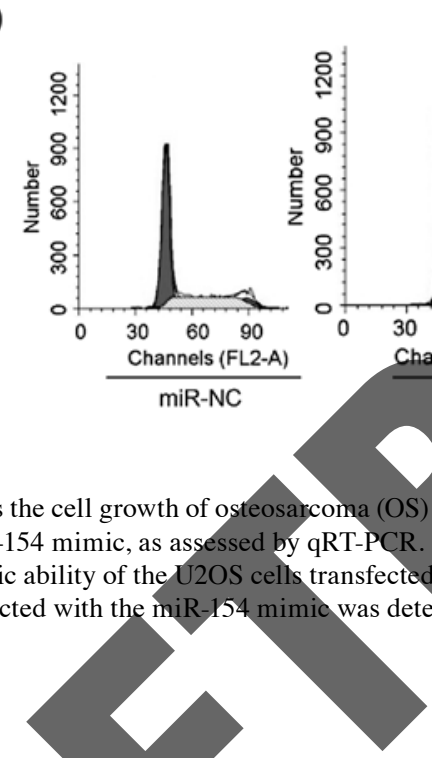

cantly decreased migration (Fig. 3A) and invasion (Fig. 3B) in the U2OS cells.

Wnt5a is a new target gene of miR-154 in osteosarcoma cells. To explore the mechanisms involved in miR-154-mediated tumor suppression, bioinformatic analysis was performed using three computational algorithms (TargetScan, miRwalk and miRanda) to predict its mRNA targets. Bioinformatic analysis showed that Wnt5a may be a direct target (Fig. 4A). To further confirm this prediction, a luciferase reporter assay was performed in U2OS cells. As shown in Fig. 4B, miR-154 significantly inhibited the luciferase activity of the wild-type (Wt) 3'-UTR Wnt5a but not the mutated (Mut) 3'-UTR Wnt5a in the U2OS cells (Fig. 4B), indicating the direct regulation of miR-154 in the 3'-UTR of Wnt5a mRNA. We next examined whether miR-154 could regulate endogenous Wnt5a expression in U2OS cells. Compared with miR-NC, endogenous Wn5a mRNA (Fig. 4C) and protein levels (Fig. 4D) were downregulated when the cells were transfected with miR-154. These results indicate that miR-154 directly binds to the 3'-UTR of Wnt5a repressing its expression.

B
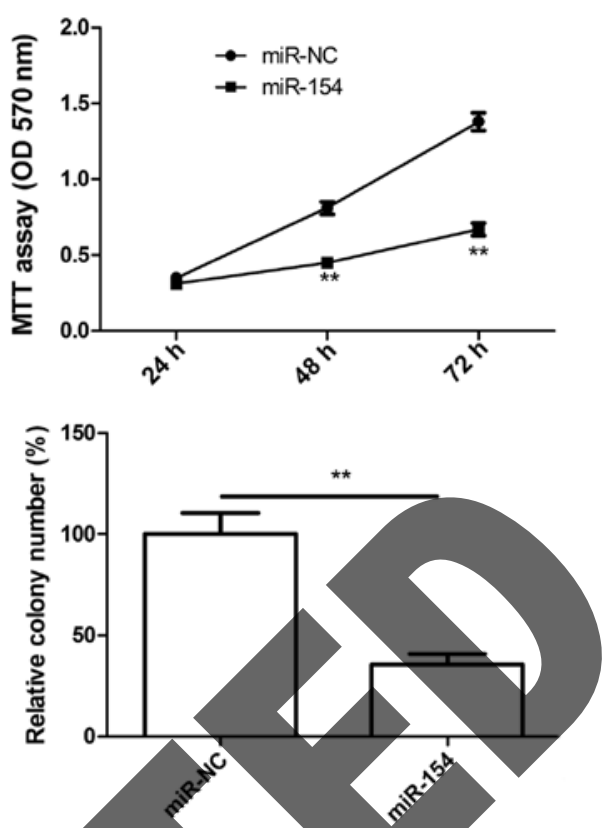

Figure 2. miR-154 inhibits the cell growth of osteosarcoma (OS) cells. (A) The expression level of miR-154 was obviously upregulated in the U2OS cells after transfection with the miR-154 mimic, as assessed by qRT-PCR. (B) Cell proliferation of U2OS cells transfected with the miR-154 mimic was determined by MTT assay. (C) Clonogenic ability of the U2OS cells transfected with the miR-154 mimic was determined by colony formation assay. (D) Cell cycle distribution of U2OS cells transfected with the miR-154 mimic was determined by flow cytometry. ${ }^{* *} \mathrm{P}<0.01$ vs. miR-NC.

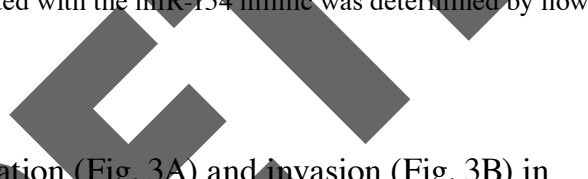

Wnt5a is inversely correlated with miR-154 in osteosarcoma. Next, we determined the expression of Wnt5a in OS samples and corresponding normal tissues. We found that Wnt5a mRNA (Fig. 5A) and protein expression levels (Fig. 5B) were upregulated compared with levels in the matched normal tissues. We also detected the Wnt5a expression in four human osteosarcoma cell lines (HOS, Saos-2, U2OS and MG-63) and normal osteoblast cells (NHOst) by western blot analysis. Wnt5a protein expression was obviously upregulated in the four OS cell lines compared with that in the normal osteoblast cells (NHOst) (Fig. 5C). Meanwhile, Wnt5a mRNA expression was inversely correlated with miR-154 expression in the OS tissues by Spearman's correlation analysis $(r=-0.935$, $\mathrm{P}<0.001$ ) (Fig. 5D).

Wnt5a overexpression attenuates the effect of $m i R-154$. To determine whether the role of miR-154 in OS is mediated by Wnt5a, U2OS cells were transfected with overexpression plasmid pCDNA3.0-Wnt5a or the vector. The efficiency of pcDNA3.0-Wnt5a transfection was determined by qRT-PCR or western blot analysis. As shown in Fig. $6 \mathrm{~A}$ and $\mathrm{B}$, the 
A

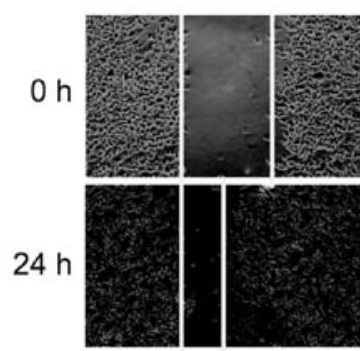

miR-NC

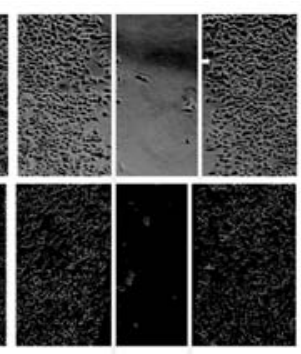

$\operatorname{miR}-154$

B

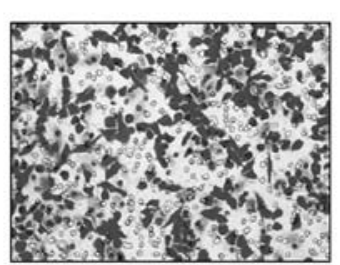

miR-NC

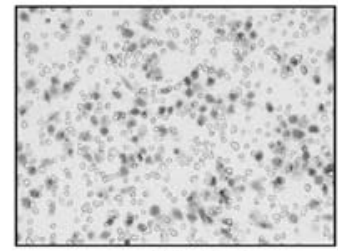

miR-154
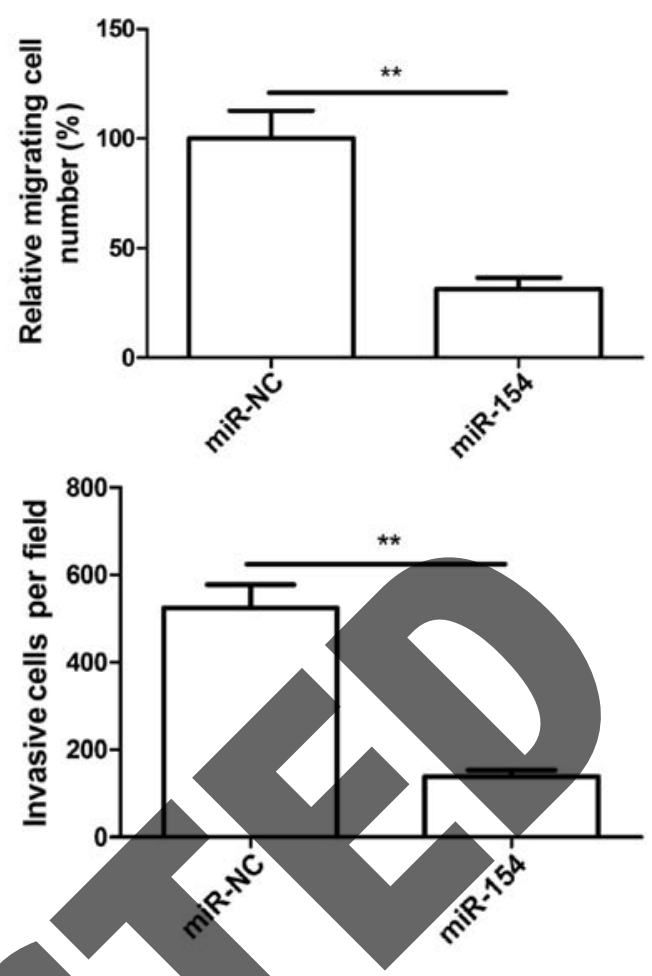

Figure 3. miR-154 inhibits the cell migration and invasion of osteosarcoma (OS) cells. (A) Cell migration of U2OS cells transfected with the miR-154 mimic was determined by wound healing assay. (B) Cell invasion of U2OS cells transfected with the miR-154 mimic was determined by invasion chamber assay. ${ }^{* *} \mathrm{P}<0.01$ vs. miR-NC.

A

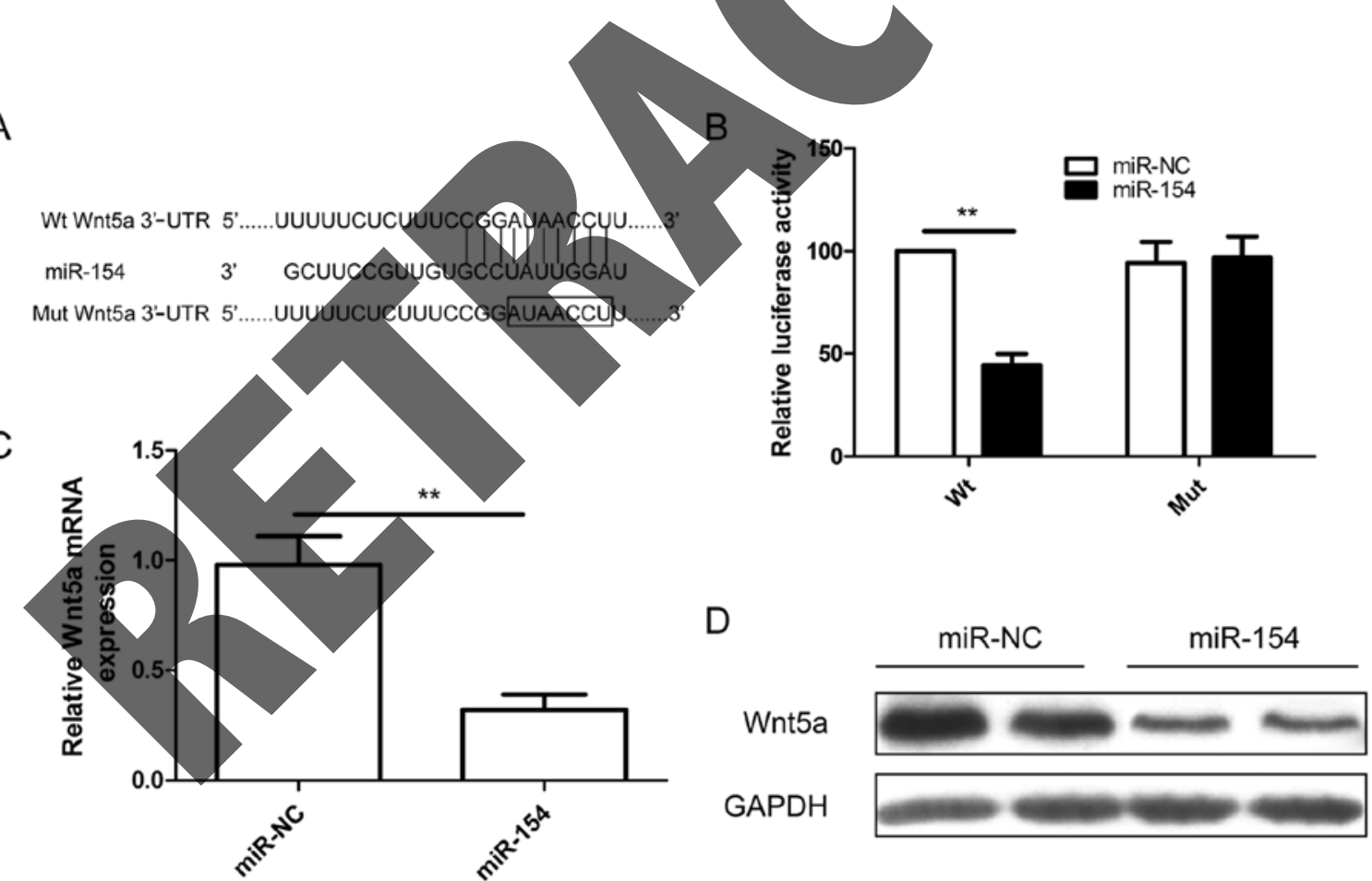

Figure 4. Wnt5a is a direct target of miR-154. (A) The putative miR-154-binding sites and mutant (Mut) 3'-UTR Wnt5a mRNA are shown. (B) Luciferase assay in U2OS cells cotransfected with miR-154 or miR-NC and a luciferase reporter plasmid containing the Wnt5a 3'-UTR (Wt) or a mutant (Mut). (C) The expression of Wnt5a mRNA was analyzed in U2OS cells transfected with the miR-154 mimic or miR-NC by qRT-PCR assay. GAPDH was used as an internal control. (D) The expression of Wnt5a protein was determined in U2OS cells transfected with the miR-154 mimic or miR-NC by western blot assay. GAPDH was used as an internal control. $\mathrm{P}<0.05 ;{ }^{* *} \mathrm{P}<0.01$ vs. miR-NC.

expression of Wnt5a at the mRNA and protein levels was significantly increased in the U2OS cells after transfection with the overexpression plasmid pCDNA3.0-Wnt5a. U2OS cells were cotransfected with miR-154 or miR-NC and a Wnt5a-overexpressing plasmid, pcDNA3.0-Wnt5a, and then cell proliferation, colony formation, migration and invasion were determined at the indicated times. The results showed that overexpression of Wnt5a dramatically reversed 
A

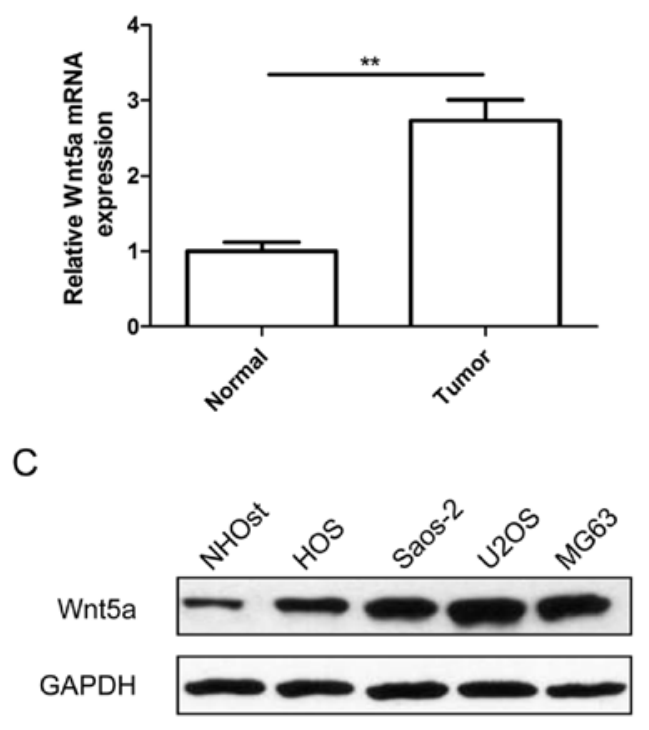

B
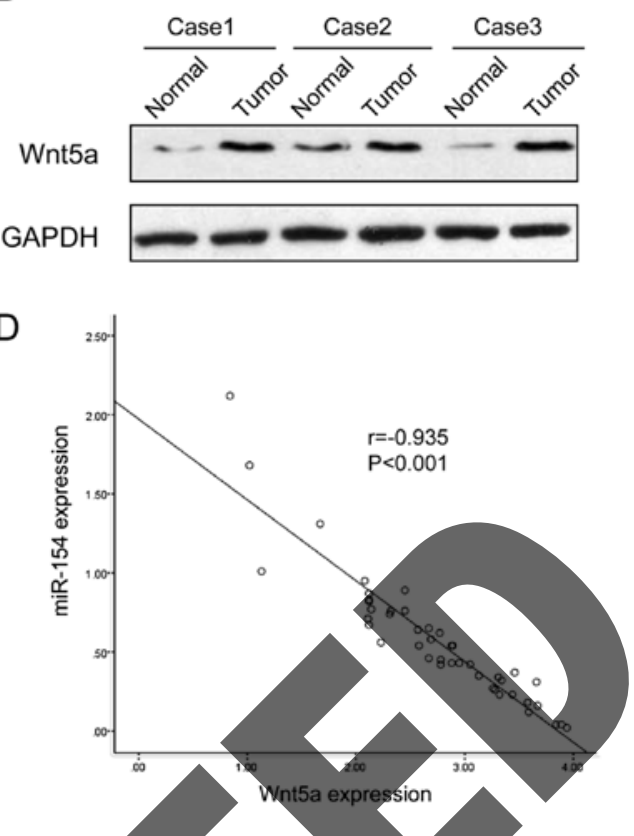

Figure 5. Wnt5a is upregulated in osteosarcoma (OS) tissues and is inversely correlated with miR-154 levels in OS tissues. (A) qRT-PCR analysis of Wnt5a mRNA expression in human OS tissue samples and their corresponding normal tissues. GAPDH was used as an internal control. ${ }^{* *} \mathrm{P}<0.01 \mathrm{vs.}$ normal tissue. (B) Western blot analysis of Wnt5a protein expression in human OS tissue samples and their corresponding normal tissues. GAPDH was used as an internal control. (C) qRT-PCR analysis of Wnt5a expression in four OS cell lines (HOS, Saos-2, U2OS and MG-63) and normal osteoblast cells (NHOst). (D) A reverse correlation between Wnt5a mRNA expression and miR-154 expression was confirmed by Spearman's correlation in OS tissues.

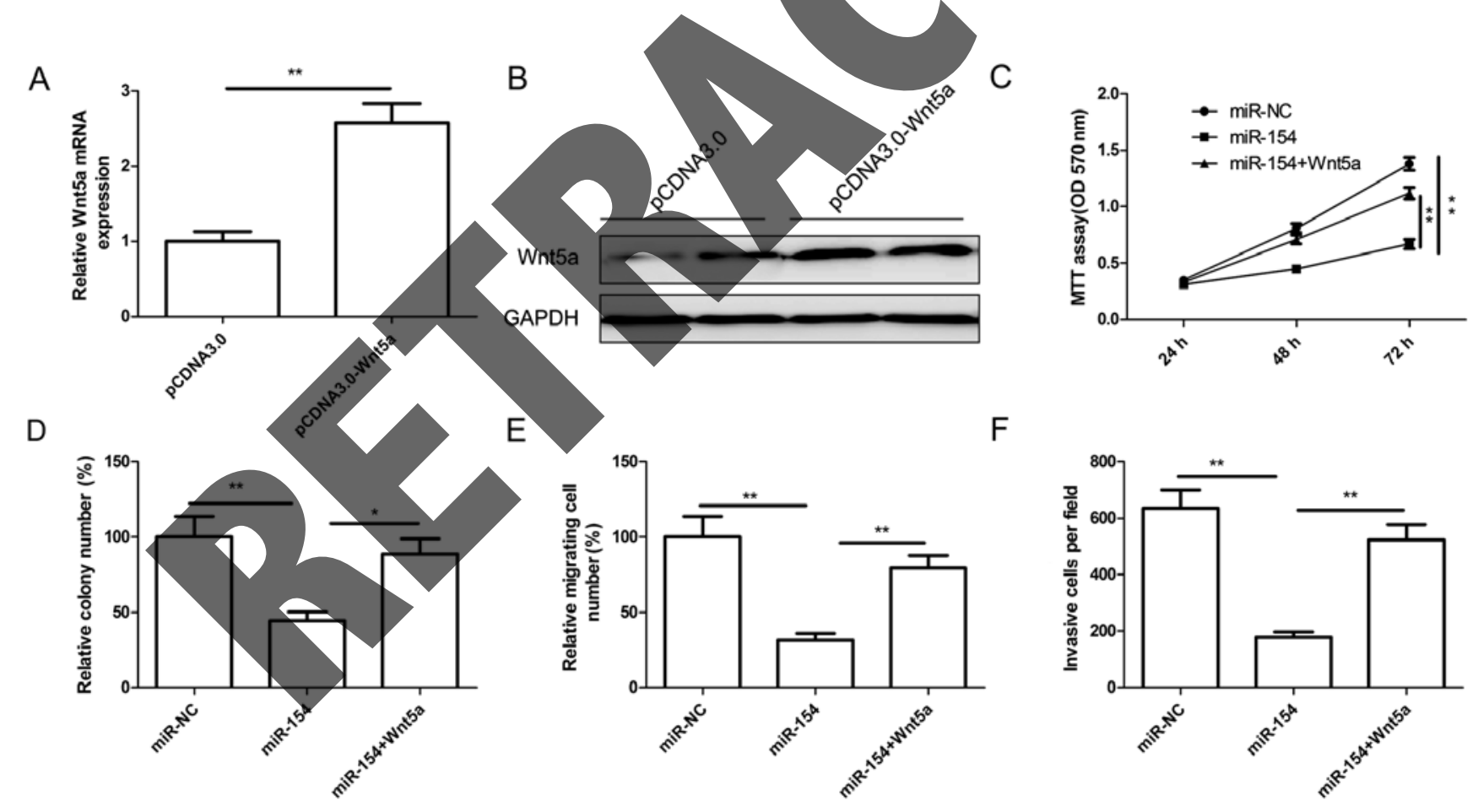

Figure 6. Wnt5a overexpression attenuates the effect of miR-154. The Wnt5a mRNA (A) and protein (B) levels were detected in U2OS cells $48 \mathrm{~h}$ after transfection with pcDNA3.0-Wnt5a or the control vector by qRT-PCR and western blot analysis, respectively. GAPDH was used as an internal control. ${ }^{* *} \mathrm{P}<0.01 \mathrm{vs}$. vector. (C-F) U2OS cells were co-transfected with miR-154 or miR-NC and Wnt5a overexpression plasmid, pcDNA3.0-Wnt5a, and then cell proliferation (C), colony formation (D), migration (E) and invasion (F) were determined in the above cells. ${ }^{*} \mathrm{P}<0.05$; ${ }^{* *} \mathrm{P}<0.01$ vs. miR-154.

the tumor-suppressive effects of miR-154 on cell proliferation (Fig. 6C), colony formation (Fig. 6D), migration (Fig. 6E) and invasion (Fig. 6F). These results suggest that miR-154 acts as a tumor suppressor in OS by targeting Wnt5a.

\section{Discussion}

miRNAs are small, endogenous non-coding RNAs that are involved in several key biological processes in tumor, such 
as tumor initiation, cell proliferation, apoptosis and metastasis (5-7). Thus, a better understanding of the underlying molecule mechanisms of miRNAs involved in tumor initiation and progression may provide a new strategy for diagnosis and therapy of various cancers including OS. Here, to the best of our knowledge, we first report that miR-154 was significantly decreased in OS tissues and cell lines, and that restoration of miR-154 suppressed OS cell proliferation, migration and invasion. Furthermore, Wnt5a was confirmed as a direct miR-154 target and its expression was inversely correlated with miR-154 expression in OS tissues. Of note, overexpression of Wnt5a substantially reversed the tumor-suppressive effects of miR-154 in regards to OS cell proliferation, migration and invasion. These results may contribute to the understand of the role of miRNAs in OS and to identify a novel potential therapeutic target for OS treatment.

miR-154, located on human chromosome $14 q 32$, is frequently downregulated in several types of cancers, including prostate (17), breast (18), liver (17), non-small cell lung (19), colorectal (20) and thyroid cancers (21). It has been reported that miR-154 functions as a tumor suppressor in several types of cancers by targeting several oncogenes (17-23). Consistent with these results, in the present study, we found that miR-154 expression was significantly downregulated in human OS tissues and exerted a tumor-suppressive function by targeting Wnt5a.

Wnt5a, an important member of the Wnt family, is a critical transcription factor in cells (22). It has been reported that Wnt5a plays an important role in various biological processe such as cell self-renewal, differentiation, migration, invasion, proliferation and apoptosis (23). Accumulating evidence shows that Wnt5a expression is upregulated in many human tumors, such as human lung squamous cell carcinoma, melanoma, gastric, breast and prostate cancer $(24,25)$, suggesting that Wnt5a has oncogenic properties. Recently studies have shown that Wnt5a expression is upregulated in OS tissues and cell lines (26), and that downregulation of Wnt5a expression in OS cells inhibited cell growth, decreased migration and invasion, as well as reduced the transformed phenotype of OS cells in vitro (27), suggesting that Wnt5a acts as an oncogene in OS and promotes the tumorigenesis of OS. In addition, Wnt5a was identified as a target of severa miRNAs, including miR-26a (28), miR-217 (29), miR-590-5p (30) and miR-374 (31). Here, we confirmed that miR-154 directly targets Wnt5a by a luciferase reporter assay, qRT-PCR and western blot analysis. We also found that Wnt5a expression was upregulated in OS tissues and cell lines, and its mRNA expression was negatively correlated with miR-154 expression in OS tissues. Of note, Wnt5a substantially reversed the tumor-suppressive effects of miR-154 on OS cells. Collectively, these findings suggest that miR-154 functions as a tumor suppressor partially by targeting Wnt5a.

Taken together, the results presented here first demonstrate that the miR-154 expression level was decreased in OS tissues and cell lines, and that restoration of miR-154 inhibited cell proliferation, migration and invasion. Moreover, we identified Wnt5a as a crucial target gene of miR-154, and found that Wnt5a expression was inversely correlated with miR-154 expression in OS tissues. Restored expression of Wnt5a weakened miR-154-mediated suppression of tumor progression.
Taken together, these findings suggest that miR-154 functions as a tumor suppressor in OS by partially suppressing Wnt5a expression.

\section{References}

1. Mirabello L, Troisi RJ and Savage SA: Osteosarcoma incidence and survival rates from 1973 to 2004: Data from the surveillance, epidemiology, and end results program. Cancer 115: 1531-1543, 2009.

2. Rytting M, Pearson P, Raymond AK, Ayala A, Murray J, Yasko AW, Johnson M and Jaffe N: Osteosarcoma in preadolescent patients. Clin Orthop Relat Res 373: 39-50, 2000.

3. Ferguson WS and Goorin AM: Current treatment of osteosarcoma. Cancer Invest 19: 292-315, 2001.

4. Yang J and Zhang W: New molecular insights into osteosarcoma targeted therapy. Curr Opin Oncol 25: 398-406, 2013.

5. Fabian MR, Sonenberg $N$ and Filipowicz W: Regulation of mRNA translation and stability by microRNAs. Annu Rev Biochem 79: 351-379, 2010.

6. Guo H, Ingolia NT, Weissman JS and Bartel DP: Mammalian microRNAs predominantly act to decrease target mRNA levels. Nature 466: 835-840, 2010

7. Lu J, Getz G, Miska EA, Alarez-Saavedra E, Lamb J, Peck D, Sweet-Cordero A, Ebert BL, Mak RH, Ferrando AA, et al: MicrøRNA expression profiles classify human cancers. Nature 435: 834-838, 2005 .

8. McManus MT: MicroRNAs and cancer. Semin Cancer Biol 13: 53-258, 200

Calin GA and Croce CM: MicroRNA-cancer connection: The beginning of a new tale. Cancer Res 66: 7390-7394, 2006.

10. Yan K, Gao J, Yang T, Ma Q, Qiu X, Fan Q and Ma B: MicroRNA-34a inhibits the proliferation and metastasis of osteosarcoma cells both in vitro and in vivo. PLoS One 7: e33778, 2012

Liu LH, Li H, Li JP, Zhong H, Zhang HC, Chen J and Xiao T: miR-125b suppresses the proliferation and migration of osteosarcoma cells through down-regulation of STAT3. Biochem Biophys Res Commun 416: 31-38, 2011.

Zhang H, Cai X, Wang Y, Tang H, Tong D and Ji F: MicroRNA-143, downregulated in osteosarcoma, promotes apoptosis and suppresses tumorigenicity by targeting Bcl-2. Oncol Rep 24: 1363-1369, 2010.

13. Ziyan W, Shuhua Y, Xiufang W and Xiaoyun L: MicroRNA-21 is involved in osteosarcoma cell invasion and migration. Med Oncol 28: 1469-1474, 2011.

14. Wei R, Deng Z and Su J: miR-217 targeting Wnt5a in osteosarcoma functions as a potential tumor suppressor. Biomed Pharmacother 72: 158-164, 2015.

15. Chong Y, Zhang J, Guo X, Li G, Zhang S, Li C, Jiao Z and Shao M: MicroRNA-503 acts as a tumor suppressor in osteosarcoma by targeting L1CAM. PLoS One 9: e114585, 2014.

16. Lin SP, Youngson N, Takada S, Seitz H, Reik W, Paulsen M, Cavaille $J$ and Ferguson-Smith AC: Asymmetric regulation of imprinting on the maternal and paternal chromosomes at the Dlk1-Gt12 imprinted cluster on mouse chromosome 12. Nat Genet 35: 97-102, 2003.

17. Wang W, Peng B, Wang D, Ma X, Jiang D, Zhao J and Yu L: Human tumor microRNA signatures derived from large-scale oligonucleotide microarray datasets. Int J Cancer 129: 1624-1634, 2011.

18. Miranda PJ, Vimalraj S and Selvamurugan N: A feedback expression of microRNA-590 and activating transcription factor-3 in human breast cancer cells. Int J Biol Macromol 72: 145-150, 2015.

19. Lin X, Yang Z, Zhang P and Shao G: miR-154 suppresses non-small cell lung cancer growth in vitro and in vivo. Oncol Rep 33: 3053-3060, 2015.

20. Xin C, Zhang $\mathrm{H}$ and Liu Z: miR-154 suppresses colorectal cancer cell growth and motility by targeting TLR2. Mol Cell Biochem 387: 271-277, 2014.

21. Mian C, Pennelli G, Fassan M, Balistreri M, Barollo S, Cavedon E, Galuppini F, Pizzi M, Vianello F, Pelizzo MR, et al: MicroRNA profiles in familial and sporadic medullary thyroid carcinoma: Preliminary relationships with RET status and outcome. Thyroid 22: 890-896, 2012. 
22. Ren D, Minami Y and Nishita M: Critical role of Wnt5a-Ror2 signaling in motility and invasiveness of carcinoma cells following Snail-mediated epithelial-mesenchymal transition. Genes Cells 16: 304-315, 2011

23. Yamagata K, Li X, Ikegaki S, Oneyama C, Okada M, Nishita M and Minami Y: Dissection of Wnt5a-Ror2 signaling leading to matrix metalloproteinase (MMP-13) expression. J Biol Chem 287: 1588-1599, 2012

24. Nishita M, Enomoto M, Yamagata K and Minami Y: Cell/tissue-tropic functions of Wnt5a signaling in normal and cancer cells. Trends Cell Biol 20: 346-354, 2010.

25. McDonald SL and Silver A: The opposing roles of Wnt-5a in cancer. Br J Cancer 101: 209-214, 2009.

26. Lu BJ, Wang YQ, Wei XJ, Rong LQ, Wei D, Yan CM, Wang DJ and Sun JY: Expression of WNT-5a and ROR2 correlates with disease severity in osteosarcoma. Mol Med Rep 5: 1033-1036, 2012.
27. Zhang A, He S, Sun X, Ding L, Bao X and Wang N: Wnt5a promotes migration of human osteosarcoma cells by triggering a phosphatidylinositol-3 kinase/Akt signals. Cancer Cell Int 14: $15,2014$.

28. Zhao S, Ye X, Xiao L, Lian X, Feng Y, Li F and Li L: miR-26a inhibits prostate cancer progression by repression of Wnt5a. Tumour Biol 35: 9725-9733, 2014.

29. Wei R, Deng Z and Su J: miR-217 targeting Wnt5a in osteosarcoma functions as a potential tumor suppressor. Biomed Pharmacother 72: 158-164, 2015.

30. Shan X, Miao Y, Fan R, Qian H, Chen P, Liu H, Yan X, Li J and Zhou F: miR-590-5P inhibits growth of HepG2 cells via decrease of S100A10 expression and inhibition of the Wnt pathway. Int J Mol Sci 14: 8556-8569, 2013.

31. Cai J, Guan H, Fang L, Yang Y, Zhu X, Yuan J, Wu J and Li M: MicroRNA-374a activates Wnt/ $\beta$-catenin signaling to promote breast cancer metastasis. J Clin Invest 123: 566-579, 2013.

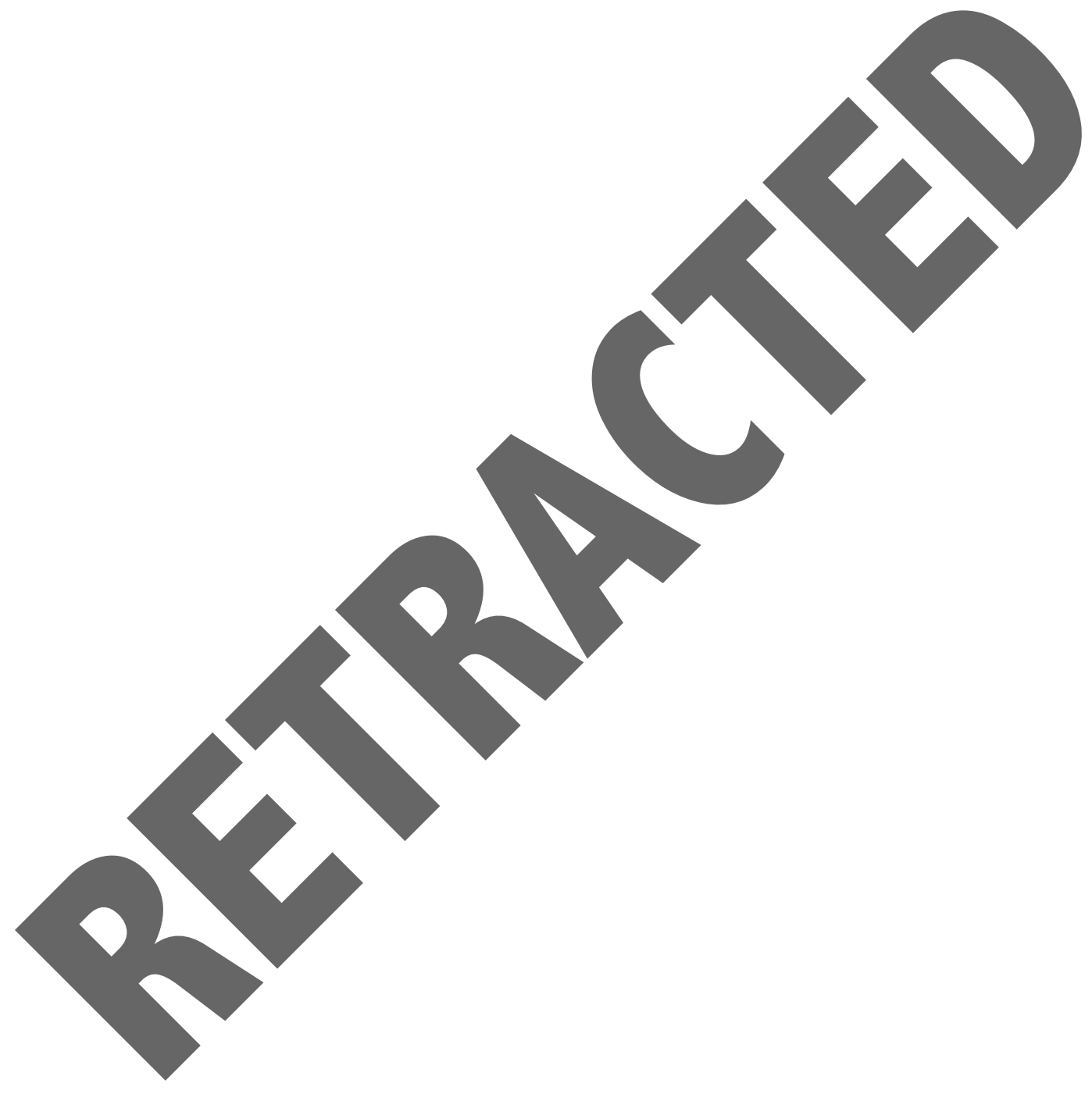

